\title{
ON THE BOREL FIELDS OF A FINITE SET
}

\section{MARLON RAYBURN}

1. Introduction. Let $X$ be a nonempty set, $\Sigma$ the complete lattice of all distinct topologies on $X$, and $\Delta$ the complete lattice of all Borel fields on $X$. It is now known [3] that $\Sigma$ is a complemented lattice. If Borel field $B$ is a topological Borel field, say generated by topology $T$, we shall indicate this by writing $B[T]$.

Consider the map $\Sigma \rightarrow \Delta$ given by $T \rightarrow B[T]$. We shall say topologies $T_{1}$ and $T_{2}$ are "Borel-equivalent," $T_{1} \sim T_{2}$, iff $B\left[T_{1}\right]=B\left[T_{2}\right]$. Then $\sim$ is an equivalence relation, so we may speak of the quotient space $\Sigma / \sim$. If $X$ is a countable space, the map is onto. If $X$ is finite, $\Delta$ is a complemented sublattice of $\Sigma$. The structure of $\Sigma / \sim$ is examined for the cases $\operatorname{card}(X)=2$ and 3 , and the structure of $\Delta$ for $\operatorname{card}(X)=4$. The method of $K$-matrices [2] is developed as a useful approach to the finite case.

\section{Development.}

Lemma. If $X$ is a countable space and $B$ a nonempty family of subsets of $X$, the following are equivalent:

(a) $B$ is a Borel field.

(b) $B$ is a closed-open topology.

(c) $B$ is a topological Borel field.

Proof. Easily, every Borel field on a countable space is a topology, and being closed under complementation, each of its sets is closedopen. On the other hand, a closed-open topology is closed under complementation, so it is a Borel field.

If $\operatorname{card}(X)=n$, (finite), let $f(n)$ be the number of distinct topologies that can be defined on $X$. Let $g(n)$ be the number of distinct Borel fields that can be defined on $X$.

Corollary. If $1<n$, then $g(n) / f(n)<1$.

It can readily be found that $g(1)=1, g(2)=2, g(3)=5$, and $g(4)$ $=15$. Krishnamurthy [2] obtained the following values for $f: f(1)=1$, $f(2)=4, f(3)=29, f(4)=355$. As an upper bound on $f(n)$, he finds that $f(n) \leqq 2^{n(n-1)}$. Below, we shall note that the square root of this number is an upper bound on $g(n)$. Krishnamurthy uses what we shall here call " $K$-matrices."

Received by the editors April 5, 1967. 
Let $\operatorname{card}(X)=n$. A " $K$-matrix" is an $n \times n$ matrix $\left(a_{i j}\right)$ where for all $i, j, a_{i j}=0$ or 1 , for all $i, a_{i i}=1$, and for all $i, j:\left(a_{j i}=1\right)$ implies $\left[\left(a_{i k}=1\right)\right.$ implies $\left.\left(a_{j k}=1\right)\right]$.

A correspondence between the set of all $n \times n K$-matrices and the family of all distinct topologies on $X$ is obtained by taking the $i$ th row $R_{i}=S\left(x_{i}\right)$ as the smallest open neighborhood of $x_{i}$, determined by $a_{i j}=1$ iff $x_{j} \in S\left(x_{i}\right)$. The final condition on $K$-matrices then reads: if $x_{i} \in S\left(x_{j}\right)$, then $S\left(x_{i}\right) \subseteq S\left(x_{j}\right)$. It is a standard exercise [1, Chapter 1 , Exercise B] that the filters generated by the $S\left(x_{i}\right)$ subject to this condition, correspond to a unique topology and that this correspondence is $1-1$ and onto. Hereafter, we shall often identify a topology $T$ with its $K$-matrix $\left(a_{i j}\right)$.

Theorem. Let topology $T$ have K-matrix $\left(a_{i j}\right)$. Then $T$ is a closedopen topology iff $\left(a_{i j}\right)$ is symmetric (with respect to the main diagonal).

Proof (Only If). Suppose for some $i, j, a_{i j}=1$ and $a_{j i}=0$. Then $x_{j} \in S\left(x_{i}\right)$ and $x_{i} \notin S\left(x_{j}\right)$. Hence $S\left(x_{j}\right)$ is a proper subset of $S\left(x_{i}\right)$. Suppose $T$ is closed-open. Then $S\left(x_{j}\right)$ is closed, so $S\left(x_{i}\right) \backslash S\left(x_{j}\right)$ is open and $x_{i} \in S\left(x_{i}\right) \backslash S\left(x_{j}\right)$. But this is a proper subset of $S\left(x_{i}\right)$, contradicting $S\left(x_{i}\right)$ the smallest open neighborhood of $x_{i}$.

$(\mathrm{IF})$. Let $\left(a_{i j}\right)$ be a symmetric $K$-matrix and suppose some set $t$ in its topology, $T$, is not closed. Then $\varnothing \neq X \backslash t$ is not open, so there exists an $x_{j} \in X \backslash t$ such that $S\left(x_{j}\right)$ is not a subset of $X \backslash t$. Hence $S\left(x_{j}\right) \cap t \neq \varnothing$. Let $x_{i} \in S\left(x_{j}\right) \cap t$ (open), so $S\left(x_{i}\right) \subseteq S\left(x_{j}\right) \cap t \subseteq S\left(x_{j}\right)$. Then $x_{i} \in S\left(x_{j}\right)$, so $a_{j i}=1$. Yet $x_{j} \notin S\left(x_{i}\right)$, so $a_{i j}=0$. Contradiction.

COROLlaRY. If $1 \leqq n$, then $g(n) \leqq 2^{n(n-1) / 2}$.

For there are that many symmetric $n \times n$ matrices whose entries are 0 and 1 , and which have 1 's on the main diagonal. It is conjectured that $\lim _{n \rightarrow \infty} g(n) / f(n)=0$.

Lemma. Let $\left(a_{i j}\right)$ be a K-matrix and define $b_{i j}=1$ iff $a_{i j}=a_{j i}=1$. Then $\left(b_{i j}\right)$ is the Borel field generated by the topology $\left(a_{i j}\right)$.

Proof. First, $\left(b_{i j}\right)$ is a $K$-matrix. Suppose for some distinct $i, j$, $b_{i j}=1$. Then $a_{i j}=a_{j i}=1$. Claim: if for any $k, b_{j k}=1$, then $b_{i k}=1$. But if $b_{j k}=1$, then $a_{j k}=a_{k j}=1$. Now $a_{i j}=1$ and $a_{j k}=1$, so $a_{i k}=1$. Moreover $a_{k j}=1$ and $a_{j i}=1$, so $a_{k i}=1$. Thus $a_{i k}=a_{k i}=1$, and $b_{i k}=1$.

Now let $T_{1}$ and $T_{2}$ have $K$-matrices $\left(a_{i j}\right)$ and $\left(b_{i j}\right)$ respectively. It is easily checked that $T_{1} \subseteq T_{2}$ iff whenever $b_{i j}=1$, then $a_{i j}=1$. $\left(b_{i j}\right)$ is symmetric by construction, so $T_{2}$ is a Borel field and is clearly the smallest Borel field containing $T_{1}$. 
Lemma. Topology $\left(a_{i j}\right)$ generates the Borel field $P(X)$, the power set, iff for all distinct $i, j$, row $R_{i} \neq$ row $R_{j}$.

Proof (Only IF). Suppose there exists some $k, p$ such that $k \neq p$ and $R_{k}=R_{p}$. Then $a_{k p}=a_{p k}=1$. Now let $\left(b_{i j}\right)$ be given by: for all $i$, $b_{i i}=1, b_{k p}=b_{p k}=1$, and $b_{i j}=0$ otherwise. Then $\left(b_{i j}\right)$ is a proper Borel field and $\left(a_{i j}\right) \subseteq\left(b_{i j}\right)$.

(IF). Suppose for all distinct $i, j, R_{i} \neq R_{j}$. If $a_{i j}=1$, then $a_{j i}=0$, else $S\left(x_{i}\right)=S\left(x_{j}\right)$ and $R_{i}=R_{j}$. Hence to find the generated Borel field, let $b_{i j}=0$ whenever $i \neq j$. But this gives the identity matrix, hence the power set.

THEOREM. Let $\left(a_{i j}\right)$ be a $K$-matrix. Then $\left|a_{i j}\right|=0$ or 1 , and $\left|a_{i j}\right|=1$ iff $\left(a_{i j}\right)$ generates Borel field $P(X)$.

Proof. The process of reducing the matrix to find its generated Borel field shows the matrix to be row equivalent to the identity matrix, and row equivalent $0-1$ matrices have the same determinant.

Corollary. Let $B$ be a Borel field with $n \times n K$-matrix $\left(a_{i j}\right)$. If $B$ contains $n-1$ singletons, then $B=P(X)$.

Proof. If $B$ contains $n-1$ singletons, then $\left|a_{i j}\right|=1$.

3. Lattice structure of $\Delta$. It is clear that the intersection of two closed-open topologies is a closed-open topology. Hence if $X$ is countable, the $\Sigma$-meet and the $\Delta$-meet of any two Borel fields coincide. On the other hand, the smallest topology containing the union of any two Borel fields is contained in the smallest closed-open topology containing that union. To see that at least in the finite case, the $\Sigma$ join coincides with the $\Delta$-join, observe that the identification of topologies with their $K$-matrices induces a lattice structure on the $K$-matrices.

A handy observation for the $\Sigma$-join of two $K$-matrices is $\left(c_{i j}\right)=\left(a_{i j}\right)$ $\bigvee\left(b_{i j}\right)$ iff $\left[\left(c_{i j}=1\right)\right.$ iff $\left(a_{i j}=1\right)$ and $\left.\left(b_{i j}=1\right)\right]$. Correspondingly for the $\Sigma$-meet of $K$-matrices, $\left(d_{i j}\right)=\left(a_{i j}\right) \wedge\left(b_{i j}\right)$ iff $\left(d_{i j}\right)$ is the smallest (least number of 1 's) $K$-matrix containing $\left(e_{i j}\right)$, where $\left(e_{i j}=1\right)$ iff $\left(a_{i j}=1\right)$ or $\left(b_{i j}=1\right)$.

THEOREM. If $X$ is finite, then $\Delta$ is a sublattice of $\Sigma$.

Proof. Let $B_{1}$ and $B_{2}$ be Borel fields with $K$-matrices $\left(a_{i j}\right)$ and $\left(b_{i j}\right)$ respectively. Let $\left(c_{i j}\right)=\left(a_{i j}\right) \bigvee\left(b_{i j}\right)$. But by the construction of $\left(c_{i j}\right)$, since $\left(a_{i j}\right)$ and $\left(b_{i j}\right)$ are both symmetric, so is $\left(c_{i j}\right)$. Hence the $\Sigma$-join of Borel fields is a Borel field. 
THEOREM. If $X$ is finite, then $\Delta$ is a complemented lattice.

Proof. Let $\left(b_{i j}\right)$ be a proper Borel field. By a previous result, if $\left(b_{i j}\right)$ contains $n-1$ singletons, it contains $n$ singletons. Hence let the number of singletons of $\left(b_{i j}\right)$ be $k$, and note $0 \leqq k \leqq n-2$. Let $\left\{x_{i}\right\}$ be a singleton not in $\left(b_{i j}\right)$ and consider the Borel field $M_{1}=\left\{\varnothing, X,\left\{x_{i}\right\}\right.$, $\left.X \backslash\left\{x_{i}\right\}\right\}$. Clearly $M_{1} \wedge\left(b_{i j}\right)=\{\varnothing, X\}$. Suppose $M_{1} \vee\left(b_{i j}\right) \neq\left(\delta_{i j}\right)$, the identity matrix. Let $p$ stand for the number of singletons in $M_{1} \bigvee\left(b_{i j}\right)$ and notice $k<p \leqq n-2$. Let $\left\{x_{j}\right\}$ be a singleton not in $M_{1} \vee\left(b_{i j}\right)$ and let $M_{2}$ be the Borel field generated by the pair of singletons $\left\{x_{i}\right\}$, $\left\{x_{j}\right\}$. Claim: $M_{2} \wedge\left(b_{i j}\right)=\{\varnothing, X\}$. [For if $\left\{x_{i}, x_{j}\right\} \in\left(b_{i j}\right)$, then $\left\{x_{i}, x_{j}\right\} \backslash\left\{x_{i}\right\}=\left\{x_{j}\right\} \in M_{1} \bigvee\left(b_{i j}\right)$.] If $M_{2} \bigvee\left(b_{i j}\right) \neq\left(\delta_{i j}\right)$, repeat the process. At each step, $M_{k} \wedge\left(b_{i j}\right)=\{\varnothing, X\}$, and for some $m$ we must have $M_{m} \vee\left(b_{i j}\right)=\left(\delta_{i j}\right)$.

It follows from a result of Steiner's [3, Theorem 1.2] that a Borel field on a countable space is a principle topology. Since Steiner establishes that every topology has a principle complement, it seems likely that $\Delta$ is a complemented lattice whenever $X$ is countable.

4. Examples. For a space of two points, the two Sierpinski (proper) topologies are both Borel-equivalent to the power set.

As an application of this, consider the following well-known result. If $\left(X, T_{1}\right)$ and $\left(Y, T_{2}\right)$ are arbitrary topological spaces, and if $f:\left(X, T_{1}\right) \rightarrow\left(Y, T_{2}\right)$ is a homeomorphism, then $f:\left(X, B\left[T_{1}\right]\right)$ $\rightarrow\left(Y, B\left[T_{2}\right]\right)$ is a Baire function. A counterexample to the converse can be found on a space of two points. Let $S_{1}$ and $S_{2}$ be the two Sierpinski topologies on $X$. Then $B\left[S_{1}\right]=B\left[S_{2}\right]=P(X)$. The identity map id: $\left(X, B\left[S_{1}\right]\right) \rightarrow\left(X, B\left[S_{2}\right]\right)$ is a Baire function, yet id: $\left(X, S_{1}\right)$ $\rightarrow\left(X, S_{2}\right)$ is not continuous.

We shall use the " $n$-basic number" notation of [2] to look at the lattice structure of $\Sigma / \sim$ for the case $n=3$, and that of $\Delta$ for $n=4$. The " $n$-basic number" for an $n \times n K$-matrix is the integer obtained by suppressing the main diagonal of the matrix and writing the remaining entries, in lexographic order of indices, as a binary number. Similarly given an $n$-basic number, its $K$-matrix can be reconstructed.

For a space of three points, the 3-basic numbers which give Borel fields are $5,18,40,63$, and 64 . Now $63[\{\varnothing, X\}]$ and $64[P(X)]$ are complementary, of course. The proper Borel fields 5, 18, and 40 are pairwise complementary. Moreover, the topologies 43 and 60 are Borel-equivalent to 40 , the topologies 30 and 51 to 18 , and topologies 15 and 53 to 5 . All the others (except 63) are Borel-equivalent to 64 .

For a space of four points, the 4-basic numbers for Borel fields and for their corresponding complements are given in the following table: 


$\quad$ Borel field
9
66
144
219
516
660
1056
1122
1581
2304
2313
2886
3504
$4095[\{\phi, X\}]$
$4096[P(X)]$

$\quad$ Is a complement of (in $\Delta$ )
$660,1122,2886,3504$
$660,1581,2313,3504$
$1122,1581,2313,2886$
$516,1056,2304$
$219,1122,2313,3504$
$9,66,1056,1122,2304,2313$
$219,660,2313,2886$
$9,144,516,660,2304,2313$
$66,144,2304$
$219,660,219,1122,1581$
$66,144,516,660,1056,1122$
$9,144,1056$
$9,66,516$
4096
4095

5. Observations. It is of some interest to note that "is a complement of" is in general neither unique nor transitive. It would be of further interest to know, in a finite space, the minimum number of complements (in $\Delta$ ) a proper Borel field can have as a function of $n$, and the minimum number of topologies in the proper equivalence classes of $\Sigma / \sim$. This latter could be used as a measure of how badly many-one the map $\Sigma \rightarrow \Delta$ is, and could give information toward our conjecture on $\lim _{n \rightarrow \infty} g(n) / f(n)$.

We have shown a characterization of those topologies Borelequivalent to $P(X)$, namely that their $K$-matrices be nonsingular. A study of those topologies ("sparse" topologies) whose generated Borel fields are proper will be made in a later paper.

\section{REFERENCES}

1. J. L. Kelley, General topology, Van Nostrand, New York, 1955.

2. V. Krishnamurthy, On the number of topologies on a finite set, Amer. Math. Monthly 73 (1966), 154-157.

3. A. K. Steiner, The topological complementation problem, Bull. Amer. Math. Soc. 72 (1966), 125-127.

UNIVERSITY OF KENTUCKY 